\title{
Image and Formula Based Gait Recognition Methods
}

\author{
Mrs.D.Sharmila MCA,Mphil, Dr.E.Kirubakaran,Member,IACSIT
}

\begin{abstract}
The term gait recognition is typically used to signify the identification of people in image sequences by the way they walk. Human gait is a valuable biometric cue that can be used for human identification besides other biometrics such as faces \& fingerprints. Gait is determined by the physical characteristics of each individual, and so is believed to be as unique to the person as a fingerprint is. Gait is relatively new and emergent behavioral biometric, that pertains to the use of an individuals walking style to determine or validate identity. Human identification using gait is a challenging computer vision task. Gait is also one of the few biometrics that can be measured at a distance, which makes it useful in surveillance applications as well. In this paper, our objective is to develop robust methods for extracting discriminate gait features automatically and passively from low-resolution video. For this, we explore two gait recognition techniques. They are Image based gait recognition and Formula based gait recognition. Both methods characterize and recognize gait by computing correspondence-free motion features from the video sequence.
\end{abstract}

\section{INTRODUCTION}

Biometric characteristics can be divided in two main classes they are physiological and behavioral. Here the first type related to the shape of the body example finger prints, face recognition, hand geometry, iris recognition and gait recognition. Second type is related to the behavior of a person like signature, keystroke dynamics and voice recognition. However, it seems unlikely that gait could be used for unique identification in the same way as other conventional biometrics. This is because gait features have poor intra-person reliability due to their dynamic nature, being dependent on various physiological, psychological and external factors (footwear, clothing, surface of walking, mood, illness, fatigue, etc.). Thus it can be used for passive identification of people in surveillance applications, unlike most other biometrics. Gait recognition is the typical term used in the computer vision community to refer to automatic extraction of visual cues that characterize the motion of a walking person in video for identification purposes. It is possible to detect and measure gait even in low resolution video.

\section{A. Contributions}

The contributions of this paper include:

* We provide two novel techniques for gait recognition. Both methods are robust to segmentation and tracking errors in that they use correspondence-free image features.
* The first method takes a image based gait recognition by computing a holistic signature of gait directly from the spatiotemporal volume of the image sequence. The method is invariant only to small variations in cadence, camera viewpoint and depth.

* The second method takes a formula based, approach by computing explicit gait parameters, viz. cadence, stride length, height, and vertical displacement.

\section{B. ASSUMPTIONS}

Both methods make the following assumptions:

- People walk with constant velocity for about 3-4 seconds.

- $\quad$ People are located sufficiently far from the camera.

- The frame rate is greater than twice the frequency of the walking.

\section{IMAGE BASED GAIT RECOGNITION}

Here we describe a gait recognition technique that takes a correspondence free holistic approach by computing the classification feature directly as a function of the spatiotemporal volume (XYT) of the walking person's image sequence. Specifically, we first compute the self-similarity plot (SSP) as the matrix of self-similarities between each pair of images of the person in the sequence. By properly aligning and scaling the SSP, we then extract normalized feature vectors that we use as input to a standard pattern classifier for gait recognition. Intuitively, the SSP encodes the properties and temporal variations of the person's silhouette shape during the walking, and thus can be regarded as a signature of gait. Furthermore, we contend that the SSP is a projection of the planar gait dynamics, provided that the person is located sufficiently far from the camera.

\section{a. Overview of Method}

A biometric system provide three functions, they are verification, Identification and Screening. Verification is somebody claims to be a person whose biometric information are already known then extract new biometric information from the person and check if those matching with the ones we have. Identification is comparing them with our database. It is a much more difficult task than verification. Screening need to check if the person belongs to a group have chosen.

Here an image sequence of a walking person (i.e. the input pattern) is processed sequentially by three main modules. A pre-processing module that segments and tracks the moving person in each frame, a feature computation/measurement module which computes the self-similarity plot and extracts normalized features from it, and a pattern classification 
module which determines the identity of the walking person based on the given features/patterns. The following figure shows the overview of this method. First stage is the data preprocessing. The goal of pre-processing data is to simplify pattern recognition problem with throwing away any important information.

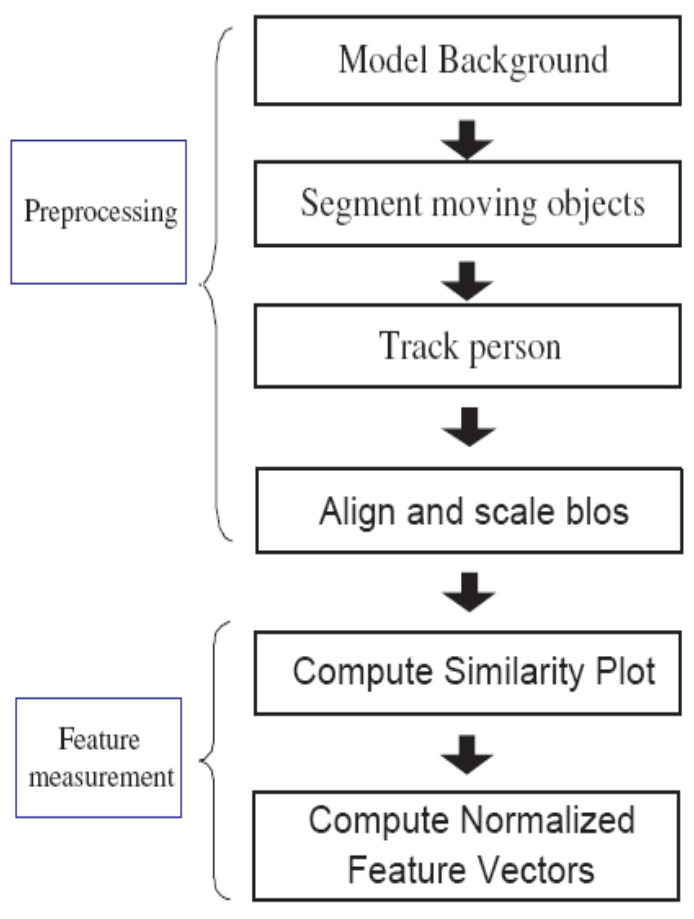

fig .1. Overview of method

Preprocessing can often reduce noise and enhance the signal. Given a sequence of images obtained from the device we detect and track the moving person, and compute the corresponding sequence of motions regions in each frame. First extract four silhouette signatures of a moving person two corresponds to inner edges and other two corresponds to outer edges of each leg.

Given a sequence of images obtained from a static camera, we detect and track the moving person, and compute the corresponding sequence of motion regions (or blobs) in each frame. Using background subtraction the detection of unusual motions can be achieved by building a representation of the scene and comparing new frames with this representation. First extract four silhouette signatures of a moving person, two corresponds to inner edges and other two corresponds to outer edges of each leg.

Each signature is normalized via spatial and temporal alignment and scaling. These normalized signatures define a spatio temporal sheet over the entire image sequence. Gait recognition is done by matching these sheets for the model gait and input gait.

b. Segmentation

To find out the segmentation errors the rectangular region for each blob is enlarged by a small radius. Motion segmentation is achieved background subtraction technique that is quite robust to lighting changes. Once detected, foreground objects are tracked in subsequent frames by simple spatial coherence, namely based on the overlap of blob bounding boxes in any two consecutive frames. Here the detection of unusual motions can be achieved by building a representation of the scene background and comparing new frames with this representation. To determine whether a tracked foreground blob corresponds to a moving person, we first compute the gait period (and hence cadence) via periodicity analysis of the width and height of the blob's bounding box. We then verify whether the computed cadence falls within some broad range of normal human cadences. We typically use the range [80,145] steps/minute, which corresponds to two standard deviations around the average cadence.

\section{c. Computing Self Similarity Plot}

This step is very important it need to choose which features to extract and how. Given a sequence of a person's N templates (i.e. cropped images) of the walking person, we compute the $\mathrm{NxN}$ of their pair wise correlations, denoted the self-similarity plot (SSP), which we shall use as the basic feature for gait recognition. Self similarity plot $S(I, J)$ is computed as the absolute correlation of each pair of templates $\mathrm{li}$ and $\mathrm{lj}$ minimized over a small search radius $\mathrm{r}$ namely.

Specifically let $w(n)$ and $h(n)$ be the width and height of the $n^{-}{ }^{\text {th }}$ image of the person. According to gait analysis literature $\mathrm{w}(\mathrm{n})$ and $\mathrm{h}(\mathrm{n})$ can be approximated as sinusoidal functions.

Define a Self Similarity Unit (SSU) as the matrix pair wise image similarities of two of the sequences. Clearly a different set of SSU's is obtained for any given starting phase $\varnothing$. We can easily show that for a sequence of $\mathrm{K}$ gait periods, the corresponding self-similarity plot contains $2(\mathrm{k}(\mathrm{k}+1)) / 2=\mathrm{k}(\mathrm{k}+1)$ contiguous SSU's .

\section{d. Normalization}

Each signature is normalized via spatial and temporal alignment and scaling. These normalized signatures define a spatial temporal sheet over the entire image sequence. Gait consists of repeated steps, and suppose each step takes $\mathrm{P}$ frames then we can divide the image sequence into contiguous segments of length $P$ frames.

The apparent size of $\mathrm{s}$ walking person varies at the frequency of gait. Specifically let w(n) and h(n) be the width and height of the $n-{ }^{\text {th }}$ image of the person.

$$
\begin{gathered}
\mathrm{w}(\mathrm{n})=\mathrm{m}_{\mathrm{w}}(\mathrm{n})+\mathrm{A}_{\mathrm{w}} \sin (\mathrm{wn}+\varnothing) \\
\mathrm{h}(\mathrm{n})=\mathrm{m}_{\mathrm{h}}(\mathrm{n})+\mathrm{A}_{\mathrm{h}} \sin (\mathrm{wn}+\varnothing)
\end{gathered}
$$

where $\mathrm{w}$ is the frequency of gait, $\varnothing$ is the phase of gait. $\mathrm{m}_{\mathrm{w}}(\mathrm{n})$ is the mean width, and $\mathrm{A}_{\mathrm{w}}$ is the amplitude of oscillation. $m_{h}(n)$ is the mean height.

Here we first computes the normalized cross-correlation of matrices template and A. The matrix A must be larger than the matrix template for the normalization to be meaningful. The values of template cannot all be the same.

\section{e. Frequency and Phase of Gait}

Obviously, we need to compute the frequency and phase of gait in order to extract the SSU's. However, there is no simple/straightforward way to estimate the phase of gait based on the width or height of the person in the image sequence. For example, the maxima/ minima of these series 
correspond to different gait poses depending on the person and the camera viewpoint.

The following figures are the input image and their conversions.

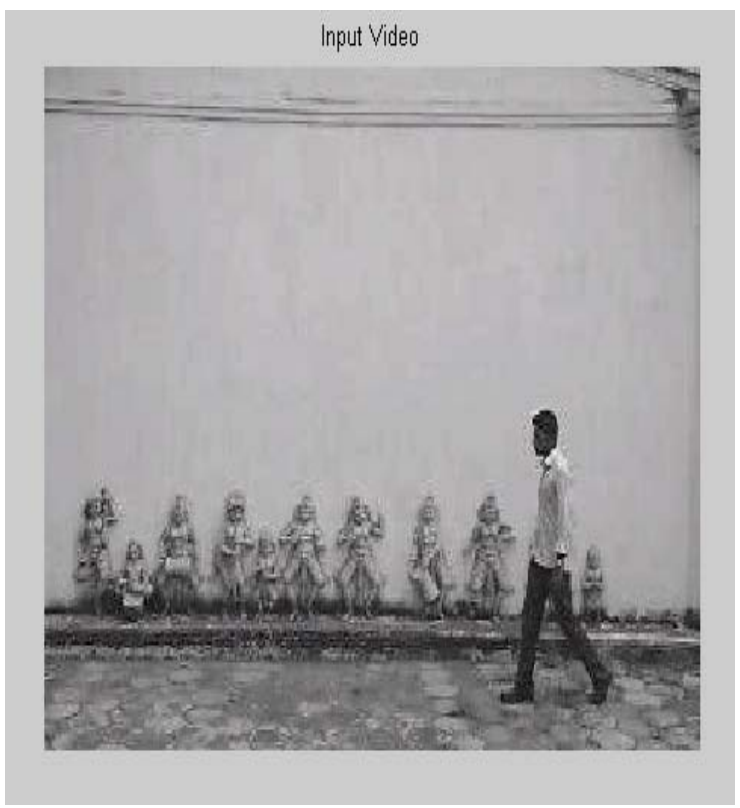

Fig 2 . Input Image

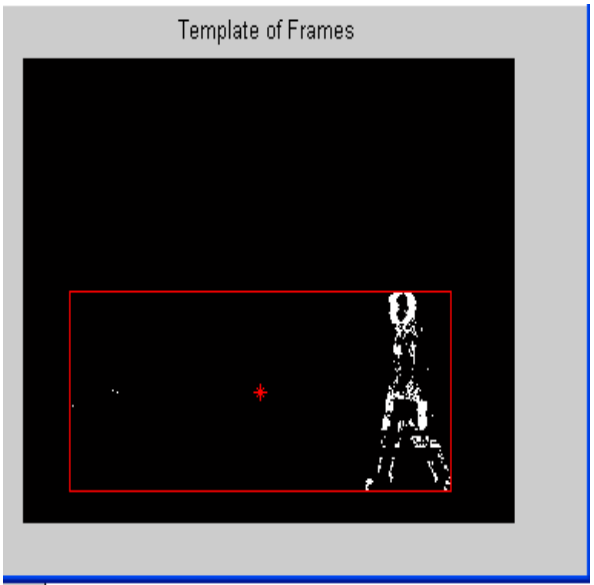

Fig 3. Template frame for input

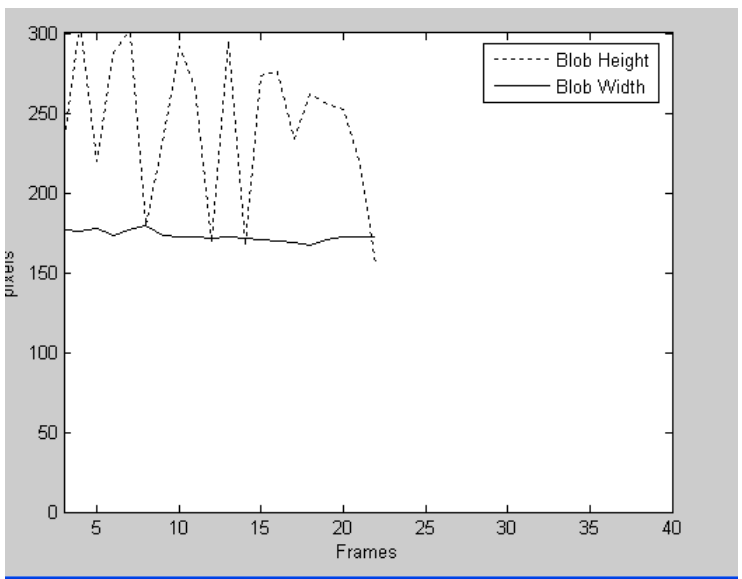

Fig 4. Blob Height and width

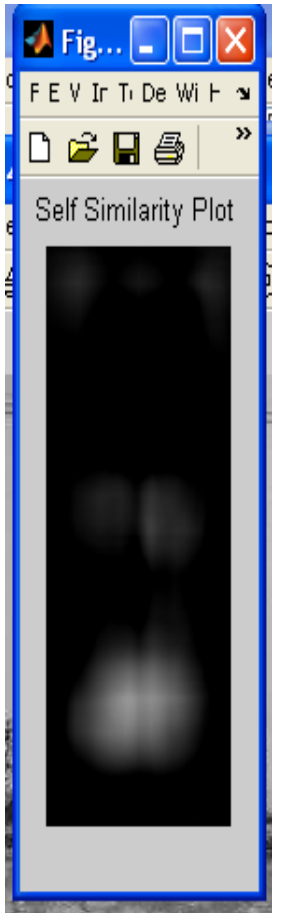

Fig 5. SSP

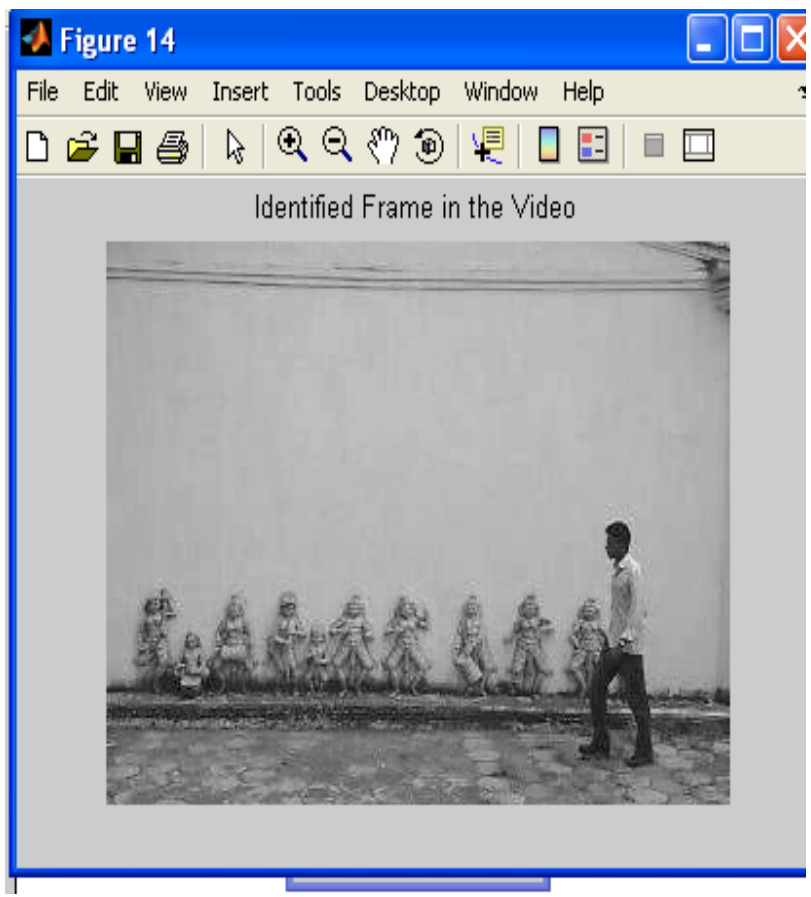

Fig .6. Identified Frame

\section{f. Classification}

The classification step is based on the use of artificial neural networks. Classification is based on the $\mathrm{K}$-nearest neighbor rule, whereby a test pattern (SSU) is assigned to the majority label of the $\mathrm{K}$ closest patterns to it in the training set where distances are measured in some space in which the patterns are represented. In the template matching approach, distances are measured in the raw SSU-space as the Euclidean distance between the two SSU's. Furthermore, to account for phase alignment errors, we shift one of the SSU's in both directions over a small radius and determine the smallest distance over all possible shifts. In the linear feature extraction approach, we compute the Euclidean 
distance in a reduced feature space of the original SSU-space.

For classification here we use Fast Fourier Transform. FFT(X) is the discrete Fourier transform (DFT)of vector X. For matrices, the FFT operation is applied to each column. For N-D arrays, the FFT operation operates on the first non-singleton dimension. $\mathrm{FFT}(\mathrm{X}, \mathrm{N})$ is the $\mathrm{N}$-point FFT, padded with zeros if $\mathrm{X}$ has less than $\mathrm{N}$ points and truncated if it has more. You can add a second argument to fft to specify a number of points $n$ for the transform $y=f f t(x, n)$ With this syntax, fft pads $\mathrm{x}$ with zeros if it is shorter than $\mathrm{n}$, or truncates it if it is longer than $n$. If you do not specify $n, f f t$ defaults to the length of the input sequence. The execution time for fft depends on the length of the transform. It is fastest for powers of two. It is almost as fast for lengths that have only small prime factors. It is typically several times slower for lengths that are prime or which have large prime factors. The inverse FFT function ifft also accepts a transform length argument.

FFT(X[],DIM)or FFT(X,N,DIM) applies the FFT operation across the dimension DIM. For length $\mathrm{N}$ input vector $\mathrm{x}$, the DFT is a length $\mathrm{N}$ vector $\mathrm{X}$, with elements $\mathrm{N}$.

\section{FORMULA BASED GAIT APPROACH}

This method extracts four static parameters, namely the body height, torso length, leg length and step length, and uses them for person identification. The first two parameters characterize the oscillation of the person's height, and the other two characterize the stride dimensions, viz. the cadence and stride length. We use the term 'apparent height' to refer to the person's height while walking, which is a time-dependent quantity, and is different from (though related to) their stature, or standing-height, which is a constant quantity. Because this height variation is an artifact of the walking movement, it is regarded as a gait variable. These features are estimated as the distances between certain body parts when the feet are maximally apart, i.e. at the double-support phase of walking. Hence, they too use stride parameters (step length only) and height-related parameters (stature, leg length and torso length) for identification. However, they consider stride length to be a static gait parameter, while in fact it varies considerably for any one individual over the range of their free-walking speeds.

\section{A. Overview of Method}

An input video sequence undergoes a sequence of processing steps to measure (i.e. estimate) the four gait variables of interest, and subsequently use them for classification/ recognition. In this method, an input video sequence is processed by three main modules, as shown in Figure 7 . The preprocessing module tracks the walking person in each frame, extracts the corresponding binary silhouette, and computes certain of its shape properties. Once the person has been tracked for a sufficient number of frames, the feature measurement module estimates the height and stride parameters from binary silhouettes. Finally, the classification module determines the person's identity via standard pattern classification, namely the K-nearest neighbor rule, in the 4-D feature space of these four gait parameters.

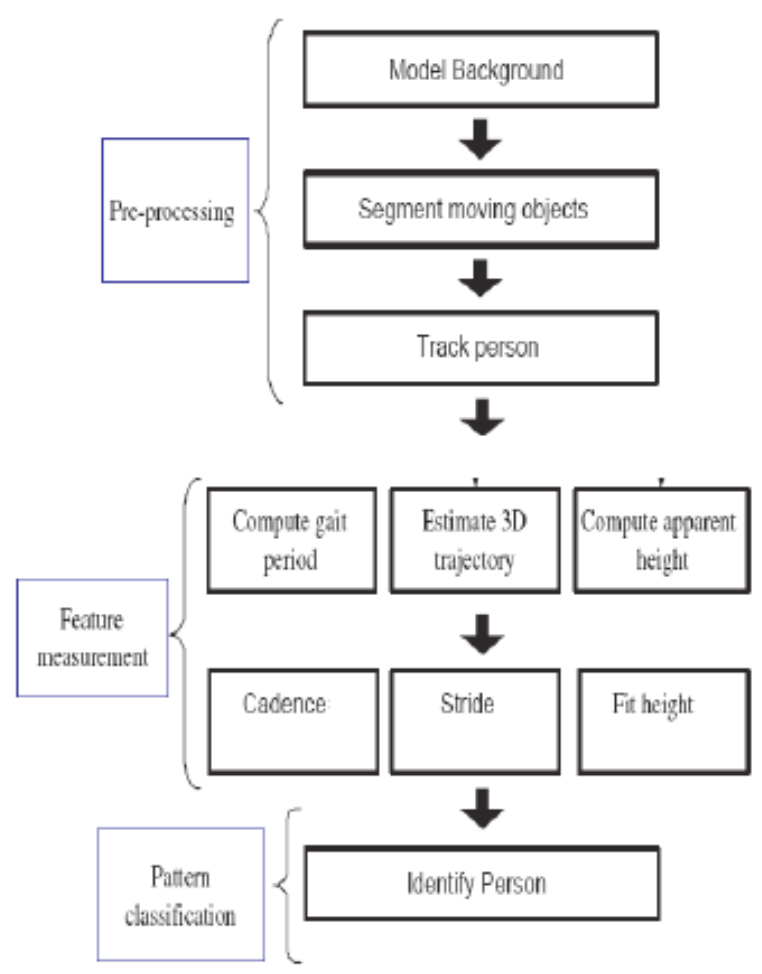

Fig 7. Overview of Method.

As shown in the block diagram of Fig. 7, the method essentially assumes the structure of a general statistical pattern classifier. An image sequence of a walking person (i.e. the input pattern) is processed sequentially and use the same method of 1.3.1 to segment and track the moving person from the background, to obtain a sequence of binary silhouettes. Then, for each binary silhouette, we compute its bounding box and locate the mid-feet point (i.e. point half-way between the two feet). The width and height of the bounding box is used to compute the gait period while the mid-feet point is used both in estimating the $3 \mathrm{D}$ position of the person on the ground and its apparent height .

\section{a. Computing Mid-feet Point}

Locating the mid-feet point in the image is done by using the midpoint of the lower edge of the silhouette's bounding box, and finds the local minimum of a horizontal projection $\mathrm{s}(\mathrm{x})$ of the binary silhouette. Specifically, for each $\mathrm{x}$ along the width of the bounding box, we define $\mathrm{s}(\mathrm{x})$ as the vertical distance at that $\mathrm{x}$ between the bottom edge of the bounding box and the lowest silhouette point contained in the lower half.

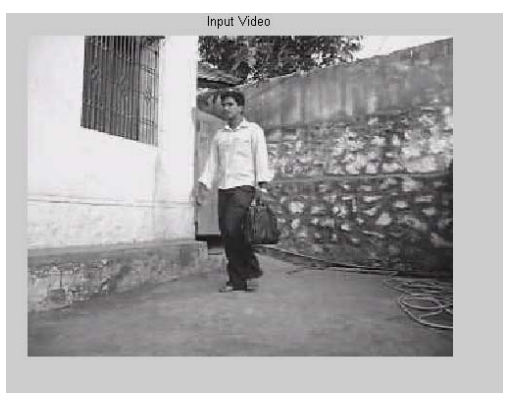

Fig 8. Input Image 


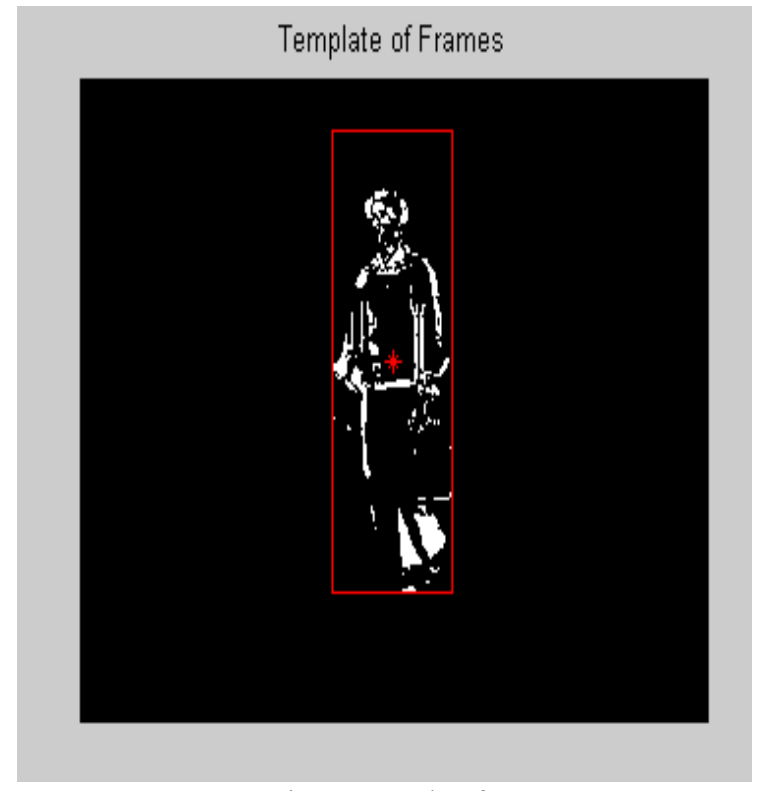

Fig 9. Template frame

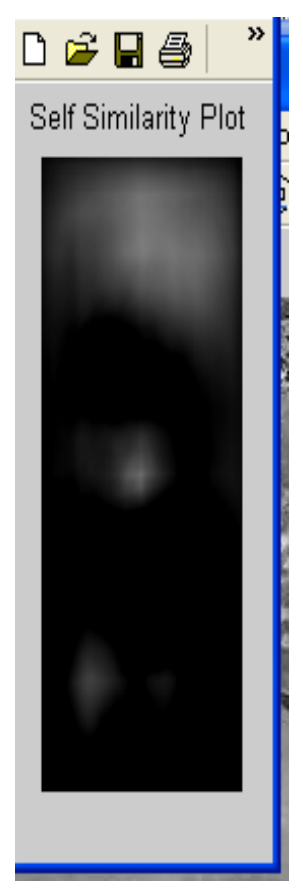

Fig 10. SSP

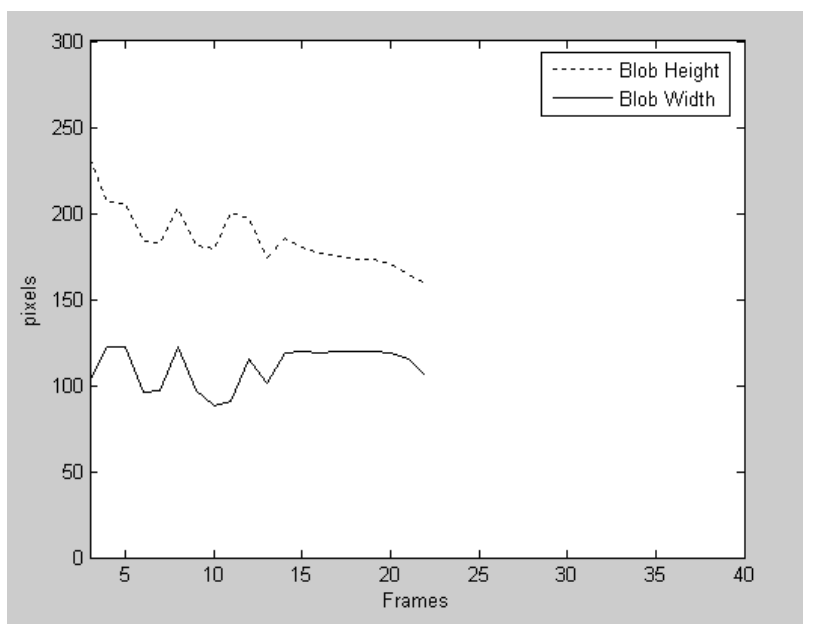

Fig 11. Blob height, width

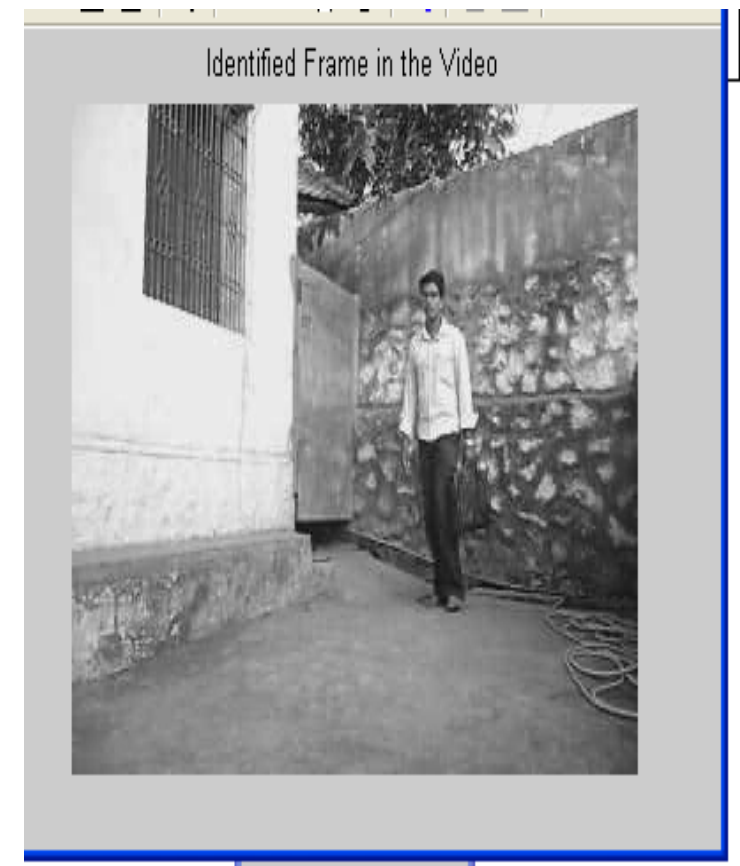

Fig 12.Identified frame

Once a person has been tracked for $\mathrm{N}$ consecutive frames, a sequence of $\mathrm{N}$ templates is then computed. Specifically, given the person's blob in each frame, we extract the (rectangular) region enclosed within the bounding box of the blob from either (1) the original color/grayscale image, (2) the foreground image, or (3) the binary image. Thus, three different types of template sequences can in fact be obtained for each person. Clearly, there are competing tradeoffs to using either type of template in measuring image similarity. (when computing the SSP).

\section{b. Estimating Period of Gait}

We use the width of the bounding box of the corresponding blob region it has used to work with our background subtraction algorithm. We estimate period, width series via the autocorrelation method which is known to be robust to non-white noise and non-linear amplitude modulations.

\section{c. Estimating 3D Trajectory}

We compute their 3D position, (Xg, $\mathrm{Yg}, \mathrm{Zg}$ ), as the 'inverse' projection of the location of their feet in the image. Furthermore, since the feet are mostly apart during walking, we use the point half-way between the two feet as the ground position, i.e. the inverse projection of the mid-feet point, denoted (xg,yg). Given the camera intrinsic (k) and extrinsic (E) matrices, and the parametric equation of the plane of motion, $\mathrm{P}: \mathrm{aX}+\mathrm{bY}+\mathrm{cZ}+\mathrm{d}=0$ in a well-defined world frame, and assuming perspective projection.

\section{d. Estimating Stride Parameters}

The basic descriptors of human locomotion are speed $\mathrm{V}$, (in meters/sec), cadence $\mathrm{C}$ (in steps/min) and stride length L(in meters/stride). Therefore, assuming nearly constant-velocity walking with period (seconds/cycle), over a distance (in meters) and steps, then and are estimated by: $\mathrm{C}=120 / \mathrm{T}, \mathrm{L}=\mathrm{W} / \mathrm{N}$, here $\mathrm{W}$ is distance in meters is steps. 


$$
\begin{aligned}
& \mathrm{C}=\frac{120}{\mathrm{~T}} \\
& \mathrm{~L}=\frac{\mathrm{W}}{\mathrm{N}}
\end{aligned}
$$

e. Estimating Height Parameters

The apparent height of a walking person can be modeled as a sinusoidal curve. The persons height in the image $h_{I}$ is estimated as the vertical distance between the head and the feet.

$$
\begin{gathered}
\mathrm{h}=\frac{\mathrm{h}_{\mathrm{f}}}{\mathrm{f}} \mathrm{Z} \\
\mathrm{C} \frac{{ }_{\mathrm{Cos} 0 \mathrm{v}-\frac{\mathrm{ya}}{\mathrm{f}} \sin 0 \mathrm{v}}^{\mathrm{f}}}{\mathrm{f}} \\
\frac{\operatorname{Cos} 0 \mathrm{v}-\frac{\mathrm{yb}}{\mathrm{f}} \sin 0 \mathrm{v}}{\mathrm{h}}
\end{gathered}
$$$$
h(t)=\mu_{h}+\alpha_{h} \sin (w t+\varnothing)
$$

$$
\begin{gathered}
\mathrm{h}=\frac{\underline{\mathrm{h}} \mathrm{Z}}{\overline{\mathrm{Cos} 0 \mathrm{v}-\frac{\mathrm{ya}}{\mathrm{f}} \sin 0 \mathrm{v}}}+\frac{\mathrm{h}_{\mathrm{I}} \mathrm{f}}{\mathrm{cos} 0 \mathrm{v}-\mathrm{yb}} \sin 0 \mathrm{v} \\
\mathrm{f}(\mathrm{t})=\mu_{\mathrm{h}}+\alpha_{\mathrm{h}} \cos (\mathrm{wt}+\varnothing)+€_{\mathrm{i}} \mathrm{t}=0,1, \ldots, \mathrm{n}
\end{gathered}
$$

\section{f. Classification}

The goal here is to build a supervised statistical pattern classifier that uses the four height and stride parameters, $\mu_{\mathrm{h}}, \alpha_{\mathrm{h}}, \mathrm{C}$, and $\mathrm{L}$ as the input feature vector to identify or verify a person in a given database. While we do not expect/claim that these features uniquely characterize a person.

Our goal is to find the single most likely value for the parameter vector given the observed data. Here we use Bayes Classifier for that and also new inputs are likely to be close to something the system has already learned means use $\mathrm{K}$ nearest neighbor non parametric pattern classifier to evaluate these features.

\section{CONCLUSIONS}

We described a novel Image based gait recognition approach that uses image self-similarity as the basic feature for classification. The method is correspondence-free and works well with low-resolution video and is robust to variation in clothing, lighting, and to segmentation and tracking errors.

And also we presented a formula based parametric approach for human identification from video using height and stride parameters of walking gait. It achieves its accuracy by exploiting the periodic nature of human walking, and computing the gait features over many steps. Significant improvement in identification performance is observed when both height and stride parameters are used than when stride parameters only are used. The method is view invariant, works with low-resolution video, and is robust to changes in lighting, clothing, and tracking errors

And also we presented a formula based parametric approach for human identification from video using height and stride parameters of walking gait. It achieves its accuracy by exploiting the periodic nature of human walking, and computing the gait features over many steps. Significant improvement in identification performance is observed when both height and stride parameters are used than when stride parameters only are used. The method is view invariant, works with low-resolution video, and is robust to changes in lighting, clothing, and tracking errors

\section{DIRECTIONS FOR FUTURE WORK}

Develop more efficient methods to compute the self-similarity plot as well as to extract classification features. And we extend this paper to determine whether the walking person has carrying an object or not. This is also related to our second formula based gait recognition.

\section{EXPERIMENTAL RESULTS}

We use sony digital camera for video input images. And use matlab for software implementation. Gait analysis commonly involves the measurement of the movement of the body in space (kinematics) and the forces involved in producing these movements (kinetics). Kinematics can be recorded using a variety of systems and methodologies.

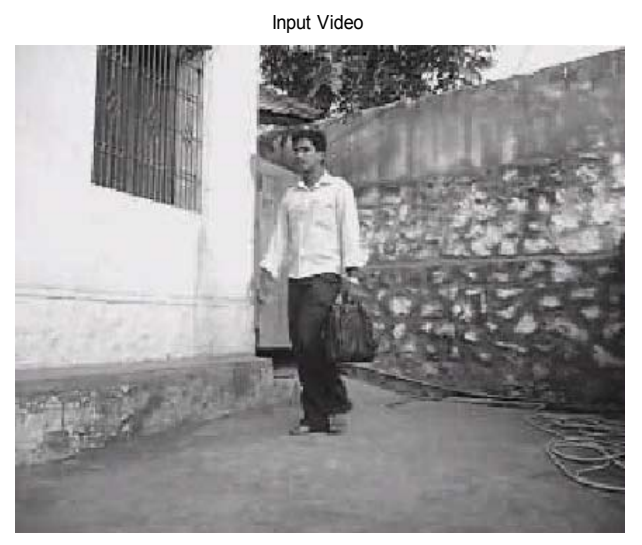

Fig 12. Input Video Sample 


\section{Self Similarity Plot}

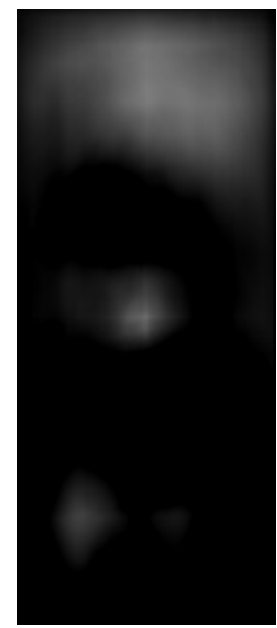

Fig 13. Sample SSP

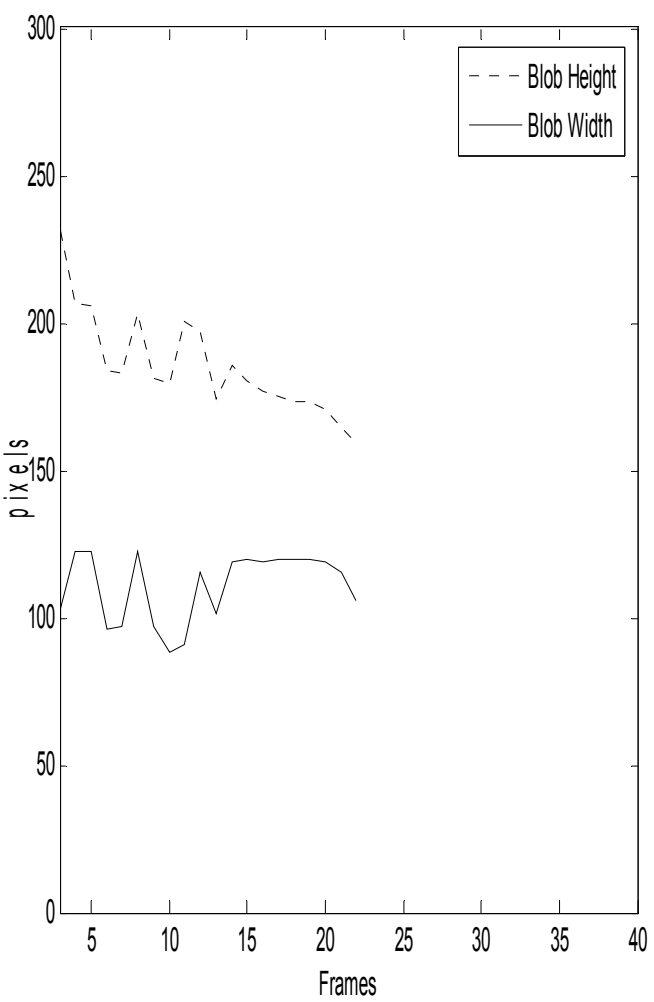

Fig 14.Blob Height and Width

\section{Identified Frame in the Video}

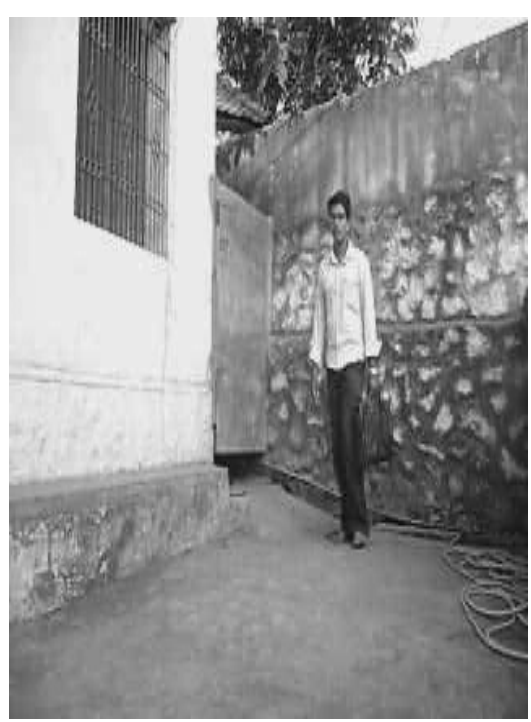

Fig 15.Sample Identified Frame

\section{REFERENCES}

[1] Anderson.A , "An introduction to Neural Networks" Addison Wesley,prentice hall,Inc, 1999.

[2] Simon Haykin, "Neural Networks", Pearson Education,1999.

[3] DonaldHearn,M.Pauline Baker,"Computer graphics",Prentice Hall inc, 1998 .

[4] Anil K.Jain,Fundamentals of digital Image Processing”,PHI,1989.

[5] Prabhat K.Andleigh, Kiran Thakrar ,"Multimesia System Design",PHI,1986.

[6] Earlgose,Richard Johnsonbaugh "Stevejost "patern recognition",PHI,1997.

[7] Christopher M.Bishop,"Neural Networks for Pattern Recognition” ,Chaman Enterprises,India,2004.

[8] Richard A.Johnson "An Introduction to Java Programming and Object oriented application Development", Thomson ,India,2007.

[9] R.K.Gupta "Audio\&video systems principles maintenance and trouble shooting" Tata 1995.

[10] Dr.Arumugam “Biomedical instrumentation” Anuradha pub ,2005.

[11] R. P.W. Duin A. K. Jain and J. MaoStatistical pattern recognition: A review. IEEE Transactions on Pattern Analysis and Machine Intelligence.

[12] N. Cuntoor A. Kale and R. Chellapa. A framework for activity based human recognition.

[13] K. Akita. Image sequence analysis of real world human motion. patternrerecognition, 17(1):73-8, 1984.

[14] P. R. Bevington and D. K. Robinson. Data Reduction and Error Analysis for the Physical Sciences. McGraw-Hill, 1992.

[15] A. Bissacco, Y. Ma A. Chiuso, and S. Soatto. Recognition of human gaits. In Proceedings of the Computer Vision and Pattern Recognition, 2001.

[16] P. Bloomfield. Fourier Analysis of Time Series: an Introduction. John Wiley and Sons, 1976.

[17] J.E. Cutting and L.T. Kozlowski. Recognizing friends by their walk: Gait perception without familiarity cues. Bulletin Psychonomic Soc., 9(5):353-356, 1977.

[18] J. Boyd. Video phase-locked loops in gait recognition. In Proceedings of the Computer Vision and Pattern Recognition, 2001.

[19] C. Bregler. Learning and recognizing human dynamics in video sequences. In Proceedings of the Computer Vision and Pattern Recognition, 1997.

First Author

D.Sharmila,Nagercoil Date of Birth: 07.05.1975. MCA,MPhil,(P.hD). MCA(Computer Applications) passed in M.K.University on 2000. Mphil (Computer Science) passed in M.K.University on 2003 B.Sc Computer Science Manonmaniam Sundaranar University on 1995. She is working as a ASSISTANT PROFESSOR (Head of Department of MCA) in Lord 
Nagar,Ramnathichenputhoor, Mylady Post.

She has published paper human Gait Recognition in Embedded for you journal vol3 issue1 page 41-43 2009. Also published papers in National and International Conferences. Doing research in Biometrics Based Authentication System.

Mrs.D.Sharmila has Member Ship in IACSIT . 